\title{
Derivadas fracionárias na modelagem do ritmo circadiano
}

\author{
Fractional derivatives in circadian rhythm modeling \\ N. M. Contessa ${ }^{1 *}$; A. De Cezaro ${ }^{2}$ \\ ${ }^{1}$ Pós-Graduação em Modelagem Computacional, FURG, 96205-900, Rio Grande-RS, Brasil \\ ${ }^{1}$ Instituto de Matemática, Estatística e Física,FURG, 96205-900, Rio Grande-RS, Brasil \\ *decezaromtm@gmail.com \\ (Recebido em 20 de abril de 2017; aceito em 22 de maio de 2017)
}

\begin{abstract}
O ritmo circadiano é responsável por diversas atividades fundamentais do organismo dos seres vivos, como produção de hormônios, atividades do sistema digestivo, a sensação de fome, de sono, etc., que funcionam de forma sincrônica. Sendo assim, é de suma importância entender os mecanismos que regem o ritmo circadiano, bem como efeitos externos que possam degradar ou acelerar processo de sincronização do ritmo circadiano.

O foco do nosso trabalho é caracterizar algumas propriedades do ritmo circadiano, levando em consideração o ciclo sono-vigília e o ciclo de temperatura corporal, bem como outros efeitos externos que possam influenciar a dinâmica do ritmo circadiano. Diferentemente do que existe na literatura, onde a modelagem da dinâmica do ciclo sono-vigília em relação a temperatura corporal é feita usando derivadas de ordem inteira, neste trabalho propomos reescrever este modelo usando cálculo de ordem fracionário. Como tal ferramenta possui propriedades que podem ser interpretadas como memória, prevemos obter um modelo que seja fidedigno a realidade, e sirva como um guia para determinar mecanismos que minimizem as alterações indesejadas causadas por interferência externas. Do ponto de vista teórico, provaremos resultados de boa colocação do modelo proposto. Apresentaremos alguns resultados numéricos que mostram o comportamento do efeito memória na dinâmica em comparação com o modelo conhecido na literatura.

Palavras-chave: ritmo circadiano, memória, derivadas de ordem fracionária
\end{abstract}

The circadian rhythm is responsible for several fundamental activities of the organism of living being such as the production of hormones, activities of the digestive system, the sensation of hunger, sleep, among other.Such actions works synchronously. Therefore, it is extremely important to understand the mechanisms that govern the circadian rhythm as well as external effects that may degrade or accelerate the synchronization process of such rhythm.

The focus of our work is to characterize some properties of the circadian rhythm, taking into account the sleep-wake cycle and the body temperature cycle, as well as other external effects that may influence circadian rhythm dynamics. Differently from what exists in the literature, where the modelling of the dynamics of the sleep-wake cycle in relation to body temperature is done using integer-order derivatives, in this work we propose to rewrite this model using a fractional order calculus. Since such a tool have properties that can be interpreted as memory, we expect to obtain a model that is reliable to reality and can be used as a guide to determine mechanisms that minimize unwanted changes caused by external interference. From the theoretical point of view, we will prove well-posedness results of the proposed model. We also provide some numerical results that show the behavior of the memory effect in the dynamics in comparison to the model known in the literature.

Keywords: circadian rhythm, memory, derivatives of fractional order

\section{INTRODUÇÃO}

Ritmo circadiano ou ciclo circadiano é um dos estudos que fazem parte da cronobiologia, área das Ciências Biológicas que tem por objetivo estudar os relógios biológicos que geram esses ritmos. Tal relógio é responsável por diversas atividades vitais do organismo dos seres vivos, funcionando como um marca-passo interno, coordenado, regulado com um período de aproximadamente 24 horas e localizado no hipotálamo. Dentre as atividades ritmicas vitais estão 
funções como, a produção de hormônios, atividades do sistema digestivo, tal como a dos rins e dos intestinos, e a sensação de fome e sono, entre outras [1,2,3,4].

Dada a diversidade de atividades do ritmicas que rege a vida dos seres vivos, modelar e entender a dinâmica do ritmo circadiano é uma maneira de detectar disfunções oriundas de descincronizações, ou eventuais efeitos externos que venham a influênciar a sincronização deste. Strogatz (1987) [1] propoz um modelo para ritmo circadiano, relacionando o ciclo sono-vigília e ciclo temperatura corporal, como a iteração de dois osciladores acoplados e cujas forças de acoplamento determinam a sincronia ou assincronia dos mesmos. A abordagem proposta por Strogatz (1987) [1] utiliza derivadas de ordem inteira para modelar a dinâmica dos dois osciladores, cujos detalhes apresentaremos na Seção 3. Como é bem sabido da teoria de diferenciação clássica, derivadas de ordem inteira reproduzem somente efeitos locais e não carregam contribuições de memória [5,6,7]. Efeitos de memória são sempre esperados em processos envolvendo o ritmo biológicos, pela própria natureza destes processos [5]. Neste trabalho estamos considerando memória como o efeito de estímulos temporários que perduram por longos períodos subsequentes. Tais efeitos são amplamente esperados na modelagem de ritmos circadianos [5].

A novidade deste trabalho está em utilizar o cálculo de ordem fracionária [6,7] para modelar a dinâmica dos osciladores proposta por Strogatz (1987) [1]. Sabendo que tais derivadas possuem propriedades de memória [6,7]. Um dos nossos objetivos é utilizar tais ferramentas para obter um modelo que seja fidedigno a realidade e sirva como um guia para determinar mecanismos que minimizem as alterações indesejadas causadas por interferência externas. Provaremos resutlados de boa colocação para o problema proposto e apresentaremos alguns resultados numéricos que determinam as características de memória na dinâmica do problema, comparada com a solução do problema proposto por Strogatz (1987) [1].

$\mathrm{O}$ artigo se divide da seguinte forma: Na Seção 2 faremos uma breve introdução as derivadas de ordem fracionária, apresentando resultados que já são amplamente conhecidos, mas que julgamos necessário para que o trabalho esteja auto-explicativo. Na Seção 3 apresentamos o modelo proposto por Strogatz $(1987,2016)$ [1,4], como uma forma simples de modelar a dinâmica de iteração entre os ciclos aqui estudados. Este serve de base para o modelo com derivadas de ordem fracionária que apresentamos na Seção 4. Nesta mesma seção apresentaremos resutlados de boa-colocação para o modelo com derivadas de ordem fracionária, bem como alguns resultados que evidenciam, de forma teórica a não-localidade de tais derivadas. Tal característica das derivadas de ordem fracionária podem ser vistos como efeitos de memória na modelagem do sistema. Uma comparação numérica entre a modelagem de Strogatz e o modelo com derivadas de ordem fracionária que estamos propondo neste trabalho podem ser encontradas na Seção 5. Na Seção 6 apresentamos algumas conclusões e perspectivas de trabalhos futuros que completam este artigo.

\section{DERIVADAS DE ORDEM FRACIONÁRIA}

Nesta seção faremos uma breve introdução ao cálculo ordem fracionária que serão utilizadas no decorrer deste trabalho. Gostariamos de deixar claro para o leitor que tal ferramente data da mesma época do cálculo desenvolvido por Newton e Leibnitz, mas que só recentemente percebeu a potencialidade de suas aplicações. Para detalhes históricos bem como de suas aplicações sugerimos ao leitor que consulte [6,7] e referências.

Iniciaremos introduzindo a derivada de ordem fracionária, segundo Riemann-Liouville e posteriormente a compararemos com a derivada de ordem fracionária segundo Caputo. Gostaríamos de deixar claro que estas não são as únicas derivadas de ordem não inteira conhecidas na literatura. No entanto, para este artigo introdutório, nós utilizaremos somente a derivada segundo Caputo. O leitor pode encontrar mais detalhes sobre as outras definições de derivadas de ordem fracionária em [6,7] e referências.

Considere $0<\alpha \leq 1$ e $\theta$ uma função suficientemente suave (detalhes sobre a suavidade da funçõa para que as derivadas de ordem fracionária introduzidas abaixo estejam bem definidas $o$ 
leitor pode encontrar em [6,7]). Definimos a derivada de ordem fracionária $0<\alpha \leq 1$ segundo Riemann-Liouville da função $\theta$ como

$$
D_{R L}^{\alpha} \theta(t)=(1 / \Gamma(\alpha-1)) d / d t \int_{a}^{t}\left(s^{-} t\right)^{-\alpha} \theta(s) d s
$$

Já a derivada de ordem $0<\alpha \leq 1$ segundo Caputo é definida por

$$
D_{C}^{\alpha} \theta(t)=(1 / \Gamma(\alpha-1)) \int_{a}^{t}(s-t)^{-\alpha} d / d s \theta(s) d s
$$

Em ambas as definições acima $\Gamma$ é a função Gama de Euler. Veja [6,7] para a definição da função Gama de Euler.

É importante observar que quando $\alpha=1$ ambas as derivadas coincidem com as derivadas de ordem inteira. Também é importante salientar que as definições de derivadas de ordem fracionária se extendem a ordens superiores, isto é, para $0<\alpha \in R^{3}$.Para detalhes consulte [6,7] e referências. Finalmente, é fácil ver que se $\theta=$ constante então $D_{R L}^{\alpha} \theta(t)$ é diferente de 0 , enquanto $D_{C}^{\alpha} \theta(t)=0$. Este fato é importante na interpretação das condições iniciais de certos sistemas dinâmicos. Veja detalhes em [6,7] e referências. Por este motivo utilizaremos a definição de derivadas de ordem fracionária de Caputo neste trabalho.

\section{MODELO DE STROGATZ}

Strogatz [1] propôs um modelo simples para o ritmo circadiano relacionando o ciclo sonovigília e temperatura corporal. Tal modelo baseia-se em dois osciladores, que são escolhidos de forma acopladas, de tal modo que, cada um acelera ou retarda o outro. Note que este modelo ignora variáveis tais como a amplitude. O objetivo do modelo não é o de representar a realidada, mas o de obter algumas características endógenas do problema.

A estrutura do modelo se apresenta da seguinte maneira: Considere dois osciladores, cujas fases serão denotados por ${ }^{\theta_{1}} \mathrm{e}^{\theta_{2}}$, representando o ciclo sono-vigília e temperatura corporal, respectivamente. Embora as fases sejam números reais, muitas vezes considerados como pontos sobre a circunferência do círculo de unidade. As equações que governam a dinâmica do modelo de fase proposto por Strogatz [1] são:

$$
\begin{aligned}
& \theta^{\prime}{ }_{1}(t)=\omega_{1}-A_{1} \cos \left(2 \pi\left(\theta_{1}-\theta_{2}\right)\right) \\
& \theta^{\prime}{ }_{2}(t)=\omega_{2}+A_{2} \cos \left(2 \pi\left(\theta_{2}-\theta_{1}\right)\right)
\end{aligned}
$$

onde $\omega_{1}>\omega_{2}$ são as frequências intríscecas, ou seja, frequências internas, e $A_{1} e A_{2}$ são as forças de acoplamento. Todos os parâmetros são considerados não-negativos.

Oscilador

Temperatura Corporal

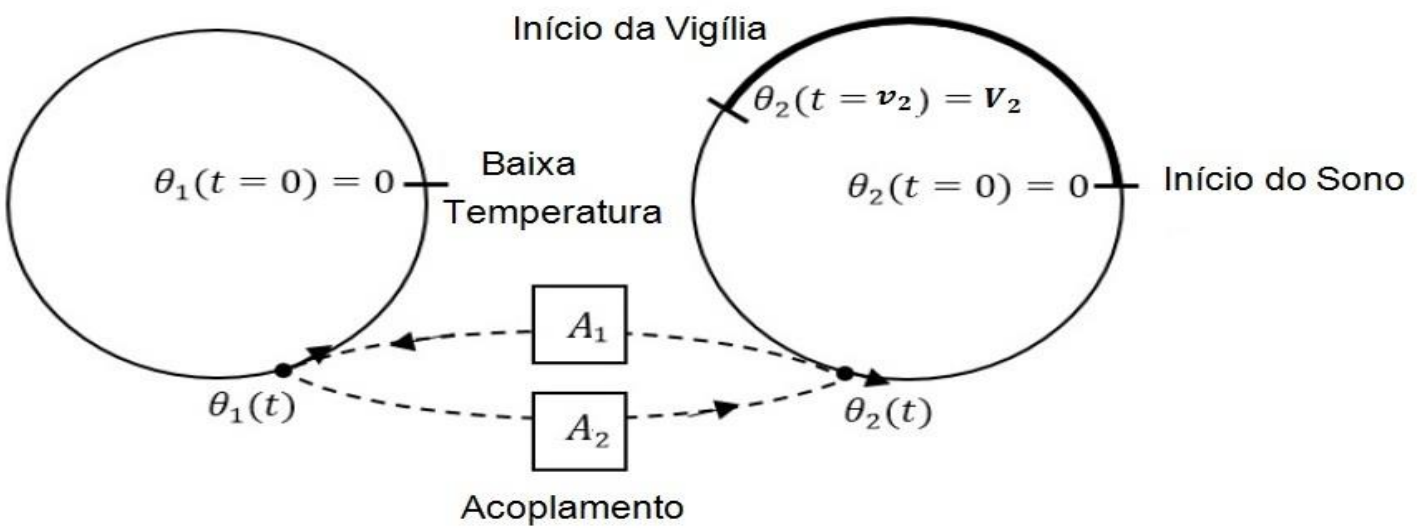

Oscilador

Sono-Vigília

Figura 1: Forma esquemática do modelo proposto por Strogatz. 
Sendo que a forma do acoplamento em que se encontram é tal que o primeiro oscilador diminui e o segundo acelera quando estão em fase (veja Figura 1), por isso os sinais trocados no sistema de equações (1).

Nesta modelagem, consideramos que o repouso é dado por uma fração $F$ do ciclo ${ }^{\theta_{1}}$ e ainda que, no início da atividade, ou seja, na vigília, temos como temperatura de referência zero. Estas duas hipóteses impõem as seguintes condições iniciais ao sistema de equações diferenciais ordinárias (1), dadas por

$$
\begin{aligned}
& \theta_{1}(0)=0, \\
& \theta_{2}(0)=0,
\end{aligned}
$$

Portanto o sistema de equações diferenciais (1) com condições iniciais (2) é um PVI.

\subsection{SINCRONIZAÇÃO E DESSINCRONIZAÇÃO NO MODELO DE STROGATZ}

Nesta parágrafo discutiremos brevemente a sincronização e a descincronização no modelo proposto por Strogatz apresentado acima. Para tal condidere a diferença de fases

$$
\Psi(t)=\theta_{1}(t)-\theta_{2}(t)
$$

Segue de (1) e (3) que

$$
\Psi^{\prime}(t)=\theta_{1}{ }^{\prime}(t)-\theta_{2}{ }^{\prime}(t)=\omega-A \cos (2 \pi \Psi(t))
$$

onde $\omega=\omega_{1}-\omega_{2}$ é a diferença de frequências intrínsecas e $A=A_{1}+A_{2}$ é a força total de acoplamento do sistema. Dada a definição de soncronização, esta ocorre quando a força de acoplamento total $A \gg \omega$, de forma que a diferença de fases seja nula, ou seja, quando $0=\Psi^{\prime}(t)$.Usando (4), temos que a sinccronização interna acontece quando a diferença de frequências

$$
\psi^{\prime}=-(1 / 2 \pi) \arccos (\omega / A)
$$

, que é a solução estável. Já a dessincronização acontece no caso contrário, ou seja quando $\omega \gg{ }^{A}$. Neste caso a diferença de fases continua a crescer quando um oscilador periodicamente supera o outro. Veremos estes casos na seção de resutlados numéricos abaixo.

\section{MODELO DE STROGATZ GENERALIZADO}

Nesta seção apresentaremos o modelo de Strogatz generalizado, que consiste em introduzir as derivadas de ordem fracionária segundo Caputo como regentes na dinâmica do sistema. Em outras palavras o sistema (1) fica escrito como

$$
\begin{aligned}
& D_{C}^{\alpha} \theta_{1}(t)=\omega_{1}-A_{1} \cos \left(2 \pi\left(\theta_{1}-\theta_{2}\right)\right) \\
& D_{C}^{\alpha} \theta_{2}(t)=\omega_{2}+A_{2} \cos \left(2 \pi\left(\theta_{2}-\theta_{1}\right)\right)
\end{aligned}
$$

onde $0<\alpha \leq 1$ e o operador de derivadas de ordem fracionária de Caputo $D_{C}^{\alpha}$ foi definido na Seção 2. As condições iniciais para a dinâmica definida na equação (6) são as mesmas que para o modelo de Strogatz, ou seja, dadas pelas condições iniciais (2).

Antes de prosseguirmos com alguns resultados numéricos, vamos garantir resultados de boa colocação dos problemas de valor inicial (6)-(2) que, no caso particular em que ${ }^{\alpha=1}$ garante a boa colocação do problema de valor inicial (1)-(2). Lembramos ao leitor que um problema é dito bem colocado, ou ainda, bem posto no sentido de Hadamard se existe uma única solução $\left(\theta_{1}(t), \theta_{2}(t)\right)$ para os sistemas em questão e que ainda tal solução dependa continuamente dos dados iniciais e dos parâmetros do sistema.

Teorema 1: O problema de valor inicial fracionário (6)-(2) é bem posto no sentido de Hadamard para qualquer $0<\alpha \leq 1$. Além disso, a solução $\left(\theta_{1}(t), \theta_{2}(t)\right)$ também depende continuamente da ordem fracionária $0<\alpha \leq 1$ da derivada. 
Demonstração: É fácil verificar que $\left[\omega_{1}-A_{1} \cos \left(2 \pi\left(\theta_{1}-\theta_{2}\right)\right), \omega_{2}+A_{2} \cos \left(2 \pi\left(\theta_{2}-\theta_{1}\right)\right)\right]$ como função das variáveis $\theta_{1} e \theta_{2}$ é uma função Lipchitiziana. Agora o resultado segue da teoria de equações diferenciais de ordem fracionária em [6,7]. Em particular, quando $\alpha=1$ temos o resultado de boa colocação do sistema proposto por Strogatz.

\section{1}

\section{EFEITOS DE MEMÓRIA}

Nesta subseção mostraremos alguns resultados que mostarm como o efeito de memória aparece na modelagem com derivadas de ordem fracionária. Em particular, mostraremos como tais efeitos de memória afetam a sincronização/descincronização do modelo de Strogatz generalizado (6) com relação ao modelo de Strogatz (1). Para perceber como os efeitos de memória agem no sistema quando aplicamos derivadas de ordem fracionária, considere dois pontos distintos $t_{1}<t_{2}$. Suponha que $\theta$ seja a solução do PVI (6)-(2) (que já sabemos que existe e é única) para $0<\alpha \leq 1$ e vamos escrever, por simplicidade $f(\theta(t), t)$ a função do lado direito da igualdade em (6). Então temos que $\theta\left(t_{2}\right)-\theta\left(t_{1}\right)=(1 / \Gamma(\alpha)) \int_{0}^{t_{2}}\left(t_{2}-t\right)^{\alpha-1} f(\theta(t), t) d t-(1 / \Gamma(\alpha)) \int_{0}^{t_{1}}\left(t_{1}-t\right)^{\alpha-1} f(\theta(t), t) d t$ $=(1 / \Gamma(\alpha)) \int_{0}^{t_{1}}\left[\left(t_{2}-t\right)^{\alpha-1}-\left(t_{1}-t\right)^{\alpha-1}\right] f(\theta(t), t) d t-(1 / \Gamma(\alpha)) \int_{t_{1}}^{t_{2}}\left(t_{2}-t\right)^{\alpha-1} f(\theta(t), t) d t$

Note que, quando $\alpha=1$ a quantidade entre colchetes na equaçõa acima é zero e portanto toda a primeira integral é nula. Isto significa que se já conhecemos a solução da equação diferencial no ponto ${ }^{t_{1}}$, então podemos calcular a solução da equação em $t_{2}>t_{1}$ baseadas exclusivamente no valor de $\theta\left(t_{1}\right)$ e f $(\theta(t), t)$. Por outro lado, para $0<\alpha<1$, o mesmo termo entre colchetes não se anula e portanto a integral não é necessariamente nula. Isto significa que, quando queremos calcular o valor da solução em um ponto ${ }^{t_{2}}$ temos que levar em consideração o que acontece com a solução em todos os pontos no intervalo $\left[0, t_{2}\right]$. Esta característica não-local da modelagem com derivadas de ordem fracionária segundo Caputo é que pode ser considerada como efeitos de memória no sistema.

\subsection{EFEITOS DE MEMÓRIA NA SINCRONIZAÇÃO/DESSINCRONIZAÇÃO}

Nesta subseção daremos uma breve explicação de como o efeito de memória da modelagem utilizando derivadas de ordem fracionária afetam a seincronização/dessincronização para o modelo de Strogatz. Tais resultados ficarão mais claros no seção dos resultados numéricos. Por simplicidade vamos olhar somente para o caso de sincronização. Para tal, seja $0<\alpha<1$. Segue da definição de derivadas de ordem fracionária que $\Psi^{\prime}(t)=D_{C}^{(1-\alpha)+\alpha} \Psi(t)=D_{C}^{(1-\alpha)}\left(D_{C}^{\alpha} \Psi(t)\right)=(1 / \Gamma(1-\alpha)) \int_{0}^{t}(t-s)^{-\alpha} d / d s\left(D_{C}^{\alpha} \Psi(s)\right) d s$.

Agora, usando a equação (4) para $D_{C}^{\alpha} \Psi(t)$ easeguinte mudança de vatiáveis $\mathrm{T}=t^{-} s$ obtemos que $\Psi^{\prime}(t)=(2 \pi / \Gamma(1-\alpha)) \int_{0}^{t} \mathrm{~T}^{-\alpha} \operatorname{sen}(2 \pi(\Psi(t-\mathrm{T}))) \Psi^{\prime}(t-\mathrm{T}) d \mathrm{~T}$.

Desta forma termos $\Psi^{\prime}\left(t^{i}\right)=0$ no modelo de Strogatz equivale a integral acima se anular. Uma possibilidade é quando $\Psi^{\prime}(t-\mathrm{T})=0$. Ou seja, vemos um efeito de adiantamento no ponto de sicronização. Veremos isso de forma mais clara nos resutlados numéricos.

Queremos deixar claro para o leitor que dada a complexidade da integral acima não foi possível obter uma relação clara para o valor da sincronização, como no modelo de Strogatz. 


\section{RESULTADOS NUMÉRICOS E DISCUSSÕES}

Nesta seção apresentaremos alguns resultados numéricos para o sistema de equações diferenciais fracionárias (6) para os valores de $\alpha=1 ; 0,9 ; 0,7 ; 0.5$, sendo as condições iniciais dadas por (2). Note que o caso em que $\alpha=1$ estamos simulando o modelo de Strogatz.

Para a simulação numérica utilizamos uma variante do método de Adams-BashforthmMoulton para derivadas de ordem fracionária, cujo custo computacional é da ordem $N(\log (N))^{2}$, onde $\mathrm{N}$ é o número de pontos de integração no tempo no intervalo $[0, T]$. Para detalhes e análise de convergência, estabilidade e especificações do método sugerimos consultar veja [8] e referências.

Nos resultados numéricos que seguem nos deteremos a analisar os casos em que o valor dado para cada frequência equivale ao número de vezes que o ciclo sofreu grandes alterações em um dia. Em outras palavras, consideraremos $\omega_{1}$, que é a frequência do ciclo da temperatura corporal, como a quantidade que representa o número de vezes que a temperatura sofreu mudanças. Como sabemos, a temperatura aumenta gradativamente durante o dia, sendo que temos o maior pico de temperatura durante a noite. Já, ${ }_{2}$ representa a frequência do ciclo da atividaderepouso. Em nossas simulações utilizaremos ${ }^{w_{2}}$ como sendo a quantidade de vezes que uma pessoa mudou do estado de sono (dormindo) para o estado de movimento ou vigília (acordado). Como não temos acessos a dados reais, a escolha dos valores de ${ }^{\omega_{1}} e^{\omega_{2}}$ foram feitos de forma sintética, ficando a calibragem de tais parâmetros, em particular de todos os parâmetos no modelo apresentado para trabelhos futuros.

Antes de apresentarmos os exemplos numéricos gostaríamos de ressaltar mais uma vez ao leitor que o modelo apresentado bem como os gráficos que representam a solução do problema geram uma característica do ritmo circadiano e não o ritmo circadiano em si. Tais características serão o fruto de uma análise particular para cada caso.

\subsection{SIMULAÇÕES PARA O CASO SINCRONIZADO}

Nesta subseção apresentaremos algumas simulações para o caso sincronizado, de forma que a escolha dos parâmetros do modelo obedecem o descrito acima e também que $\omega<A$. Para as simulações que apresentaremos abaixo utilizaremos os seguintes valores para os parâmetros do modelo $A_{1}=5, A_{2}=4, \omega_{1}=10 e \omega_{2}=2$. Lembramos ainda que, embora tenhamos feito simulações para vários valores de $0<\alpha \leq 1$ apresentaremos somente para os casos descritos anteriormente.

A Figura 2 apresenta o resultado da simulação para o modelo de Strogatz (1)-(2) (que é equivalente a $\alpha=1$ no modelo (6)-(2)) para a escolha de parâmetros acima. Como pode ser visto a sincronização ocorre em $\mathrm{t}=0.1$, o que é compatível com a escolha de parâmetos e os resultados teóricos para o modelo de Strogatz. 


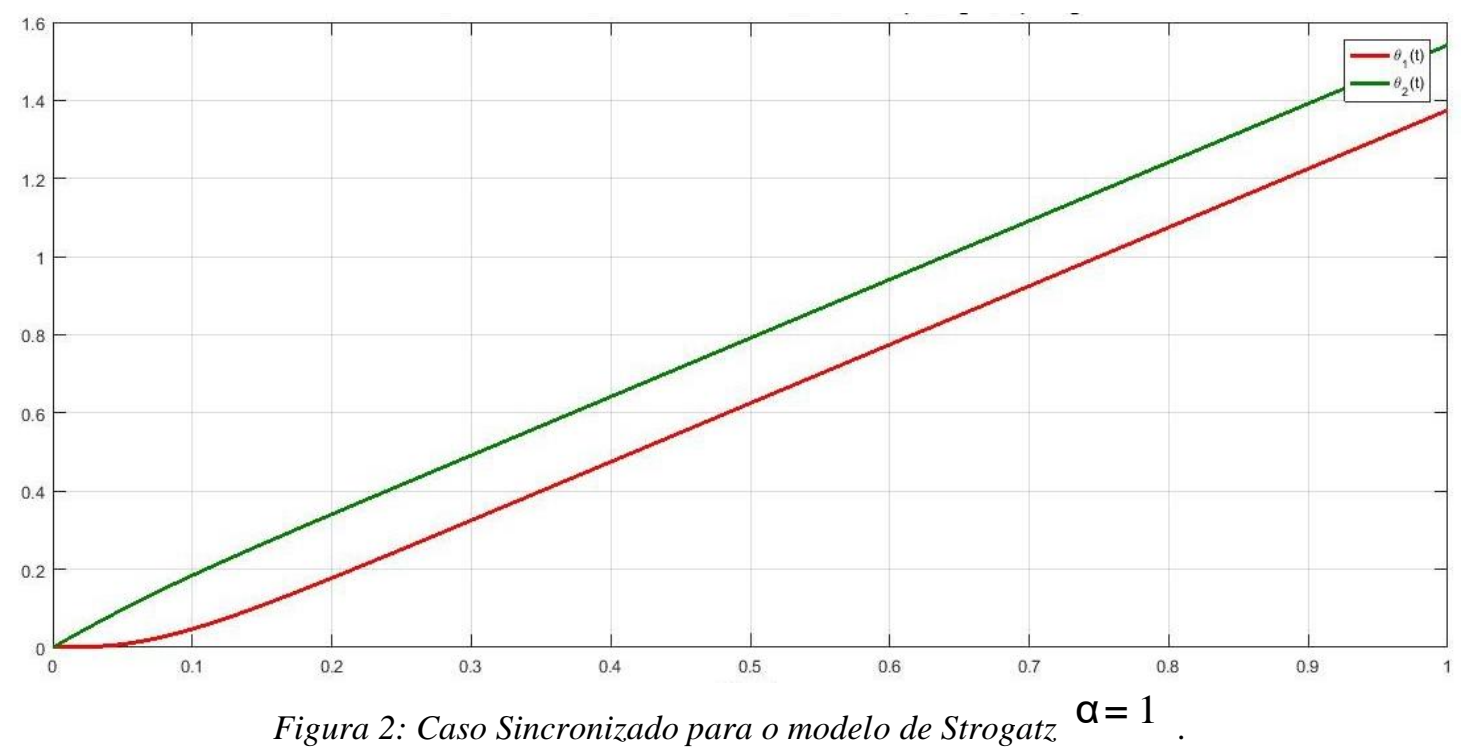

Na figura 3 apresentamos a solução do modelo de Strogatz Generalizado (6)-(2) com a ordem fracionária da derivada $\alpha=0.9$. É possível perceber que a solução diferencia pouco com a apresentada na Figura 2, mostrando que o resutlado do Teorema 1 foi respeitada na simulação numérica. Além disso, é possível notar que o ponto de sincronização foi transladado na direção da origem do sistema, conforme previsto na Subseção 3.2.

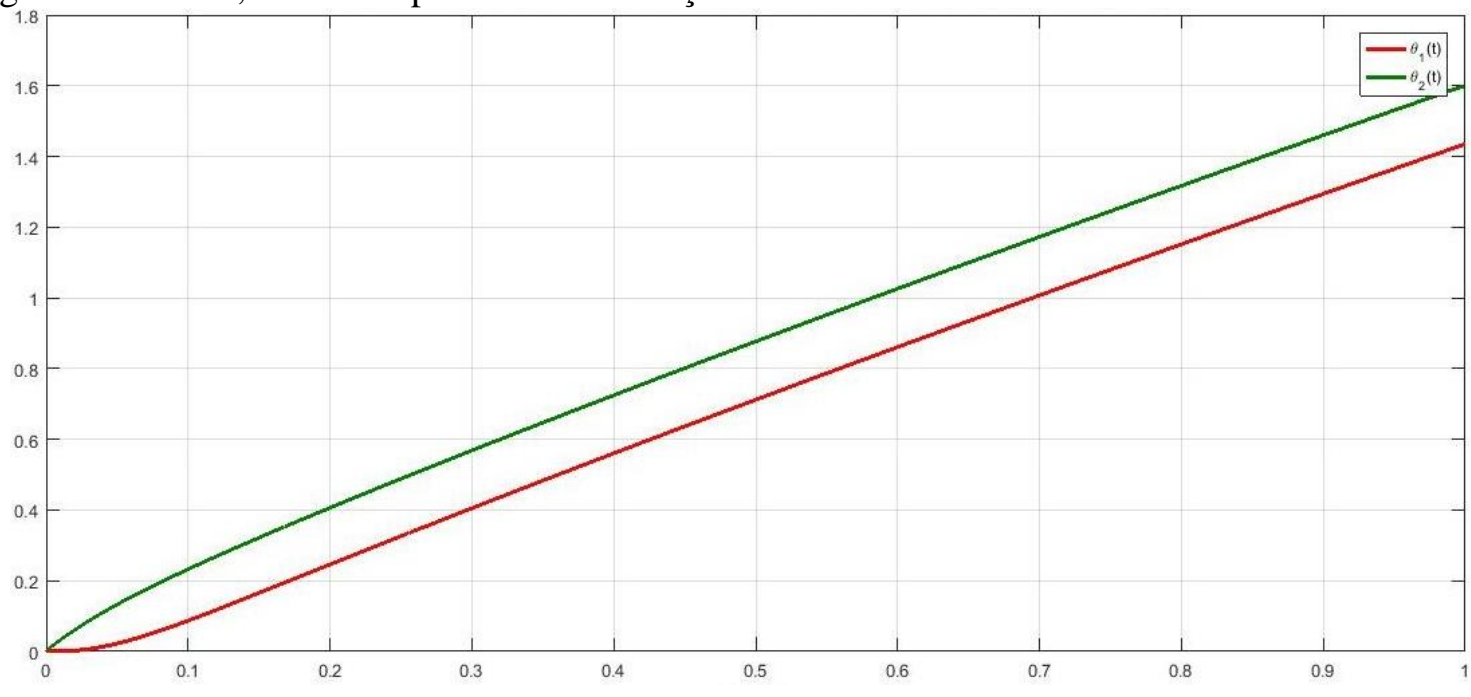

Figura 3: Caso Sincronizado modelo de Strogatz Generalizado $\alpha=0.9$

Na figura 4 apresentamos a solução do modelo de Strogatz Generalizado (6)-(2) com a ordem fracionária da derivada $\alpha=0.7$. É possível perceber que a solução apresenta características que não aparecem nas Figura 2 e 3 . Além do ponto de sincronização foi transladado na direção da origem do sistema, conforme previsto na Subseção 3.2, a solução mostra uma característica de concavidade voltada para baixo. Ambas as características podem ser consideradas como efeito de memória do sistema. 


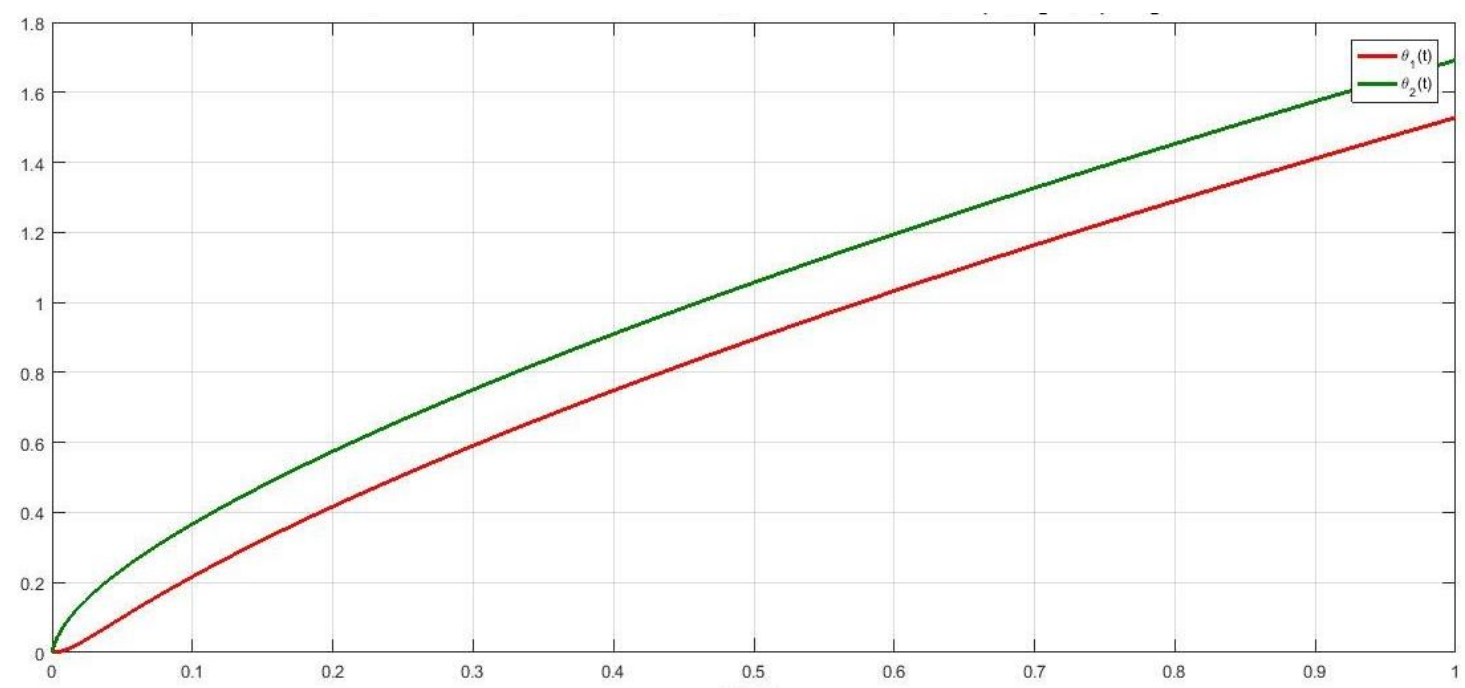

Figura 4: Caso sincronizado para modelo de Strogatz generalizado $\alpha=0.7$.

Na figura 5 apresentamos a solução do modelo de Strogatz Generalizado (6)-(2) com a ordem fracionária da derivada $\alpha=0.5$. É possível perceber que a solução apresenta características que não aparecem nas Figura 2 e 3 e as características de concavidade voltada para baixo se acentuam. Neste sendido, podemos caracterizar o modelo com esta escolha da ordem da derivada, como um modelo com muita memória. Tal padrão é esperado, dada a definição da derivada de ordem fracionária.

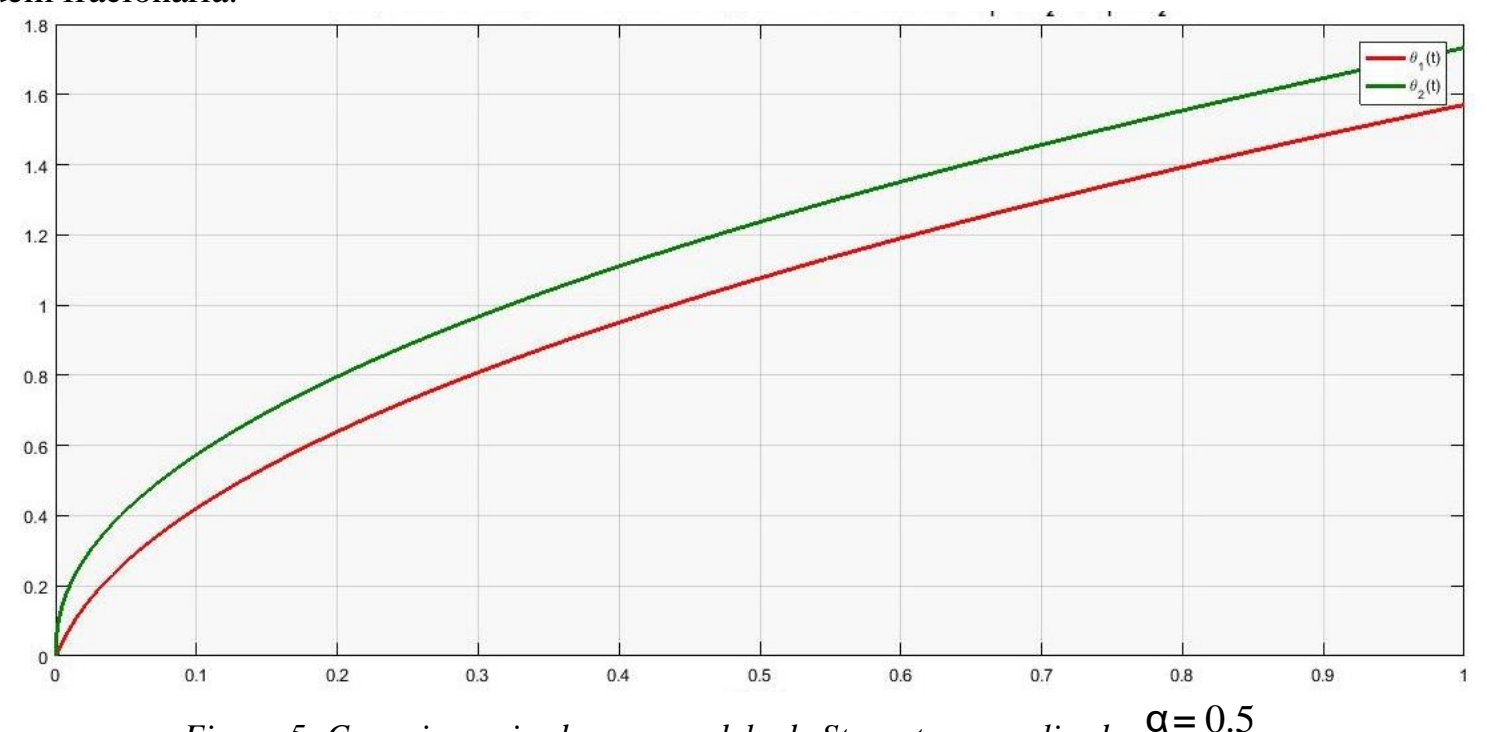

Figura 5: Caso sincronizado para modelo de Strogatz generalizado $\alpha=0.5$.

Dado os resultados numéricos acima, fica claro que a modelagem do problema utilizado derivadas de ordem fracionária apresentam um comportamento de memória do sistema. Para que as escolhas dos parâmetros e da ordem da derivda sejam feitos de maneira compatível com a realidade faz-se necessário a obtenção de dados reais e a utilização de métodos de calibragem dos parâmetros. Ambos resultados serão fruto de trabalhos futuros.

\subsection{SIMULAÇÕES PARA O CASO DESSINCRONIZADO}

Analisaremos agora o caso dessincronizado. Seguindo a definição de Strogatz, fazendo uso para os seguintes parâmetros $A_{1}=2, A_{2}=6, \omega_{1}=10 e \omega_{2}=1$ como forças de acoplamento e frequências intrínsecas, respectivamente. Desta forma temos que $A<\omega$. 
A Figura 6 apresenta o modelo de Strogatz (1)-(2) para o caso dessincronizado para a escolha de parâmetros acima. Observamos pelo gráfico apresentado na Figura 6, que desde a origem, o gráfico apresenta dessincronização. A interpretação de tal resultado é a seguinte: $O$ comportamento dos osciladores não segue um padrão, se apresenta de modo diferente, o que sugere que o sistema está em dessincronização interna.

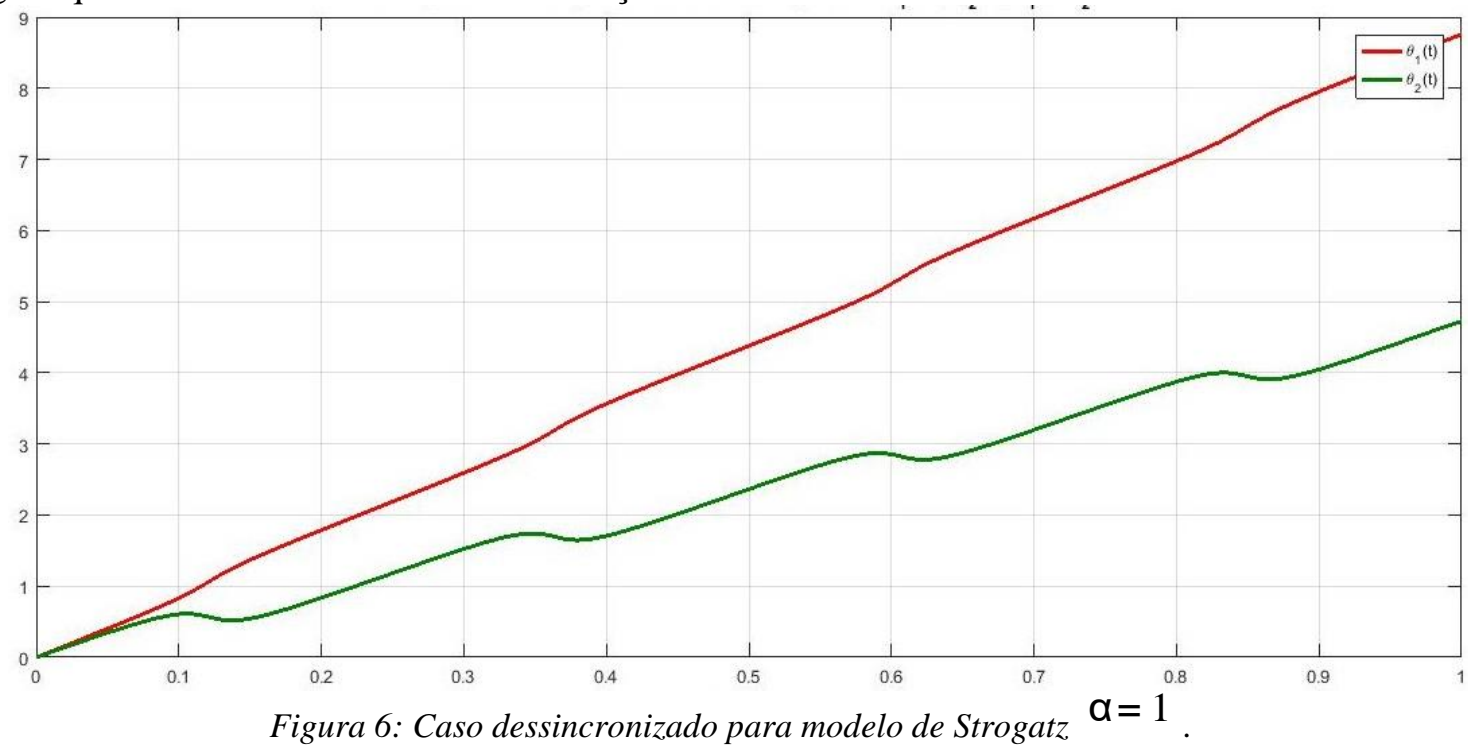

As Figuras 7, 8 e 9 representam a solução do modelo de Strogatz generalizado para $\alpha=0.9,0.7 e 0.5$, respectivamente. Notamos que a diferença entre o gráfico da solução do modelo de Strogatz e do modelo de Strogatz generalizado para apresenta diferença considerável. Os osciladores parecem procurar um padrão em determinado período mais longo quando tratamos do modelo de Strogatz generalizado quando comparado com o modelo de Strogatz da Figura 6. Observamos que este efeito é cada vez mais evidente, quanto menor o valor da derivada fracionária , como é possível observar nas Figuras 8 e 9. Tais efeitos são compatíveis com os processos de memória para os quais o modelo de Strogatz generalizado é proposto. Em outras palavras, esse padrão visualizado nos gráficos é compatível com modelos que utilizam memória tanto do ponto de vista teórico e também é o observado na prática, dando assim o suporte ao modelo proposto.

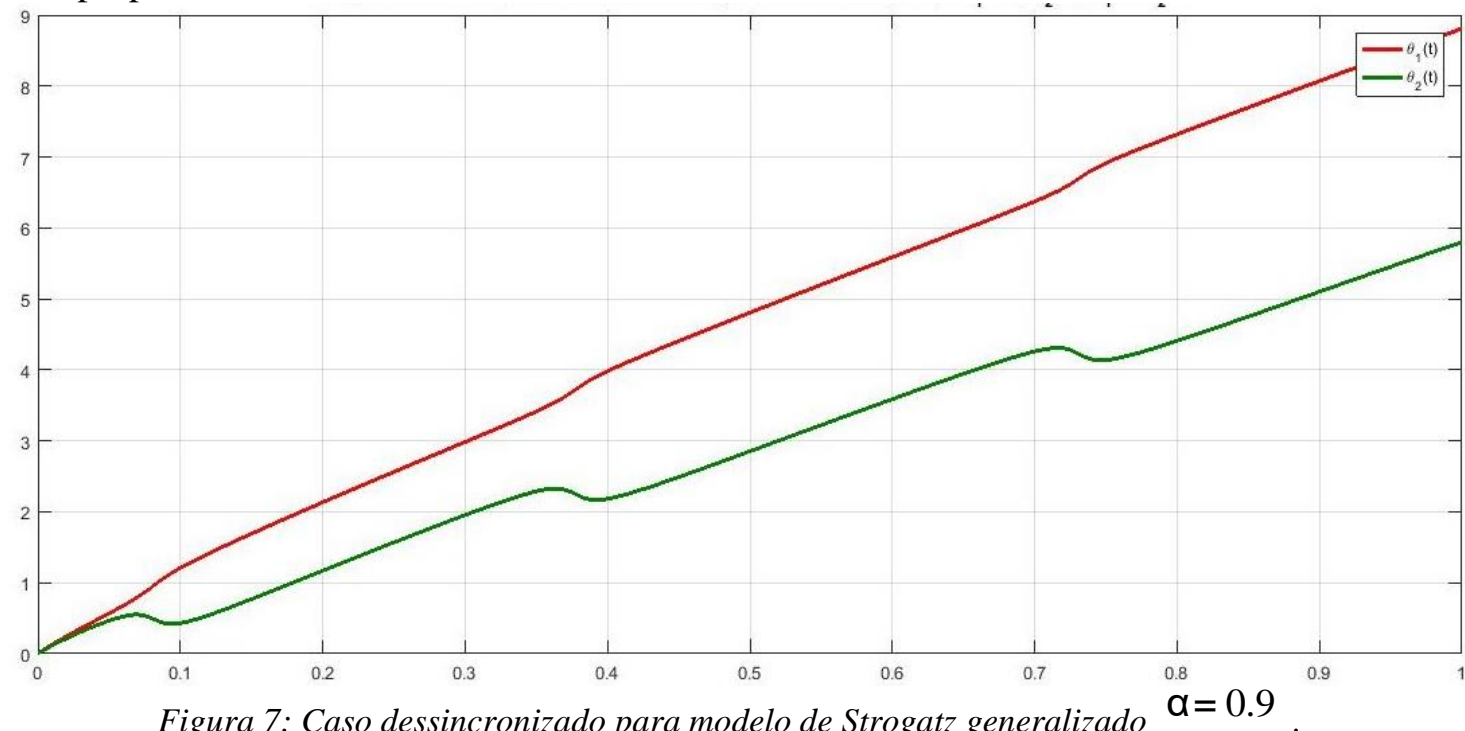

Figura 7: Caso dessincronizado para modelo de Strogatz, generalizado $\alpha=0.9$ 

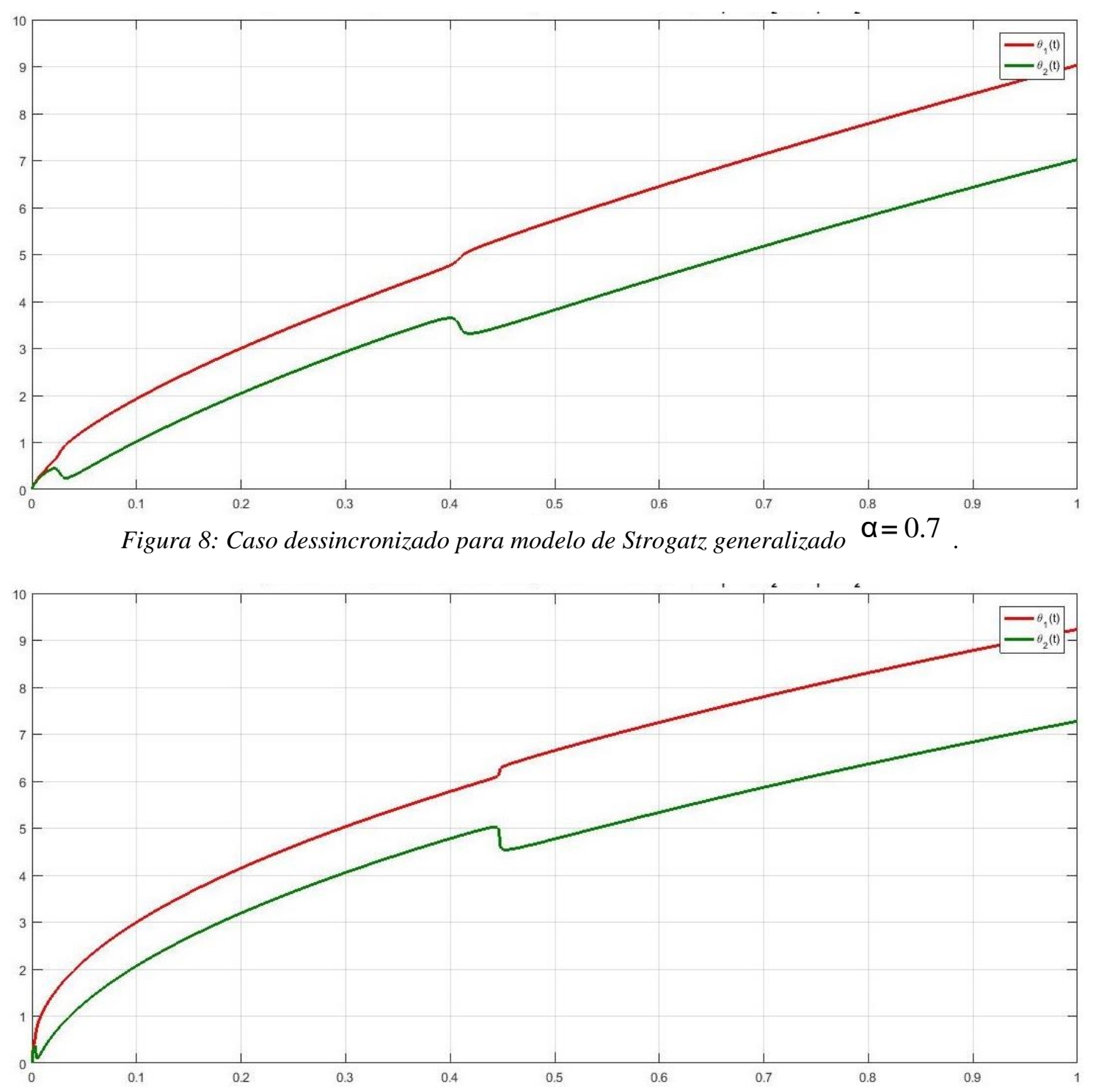

Figura 9: Caso dessincronizado para modelo de Strogatz generalizado $\alpha=0.5$.

Novamente salientamos que uma conclusão definitiva quando a ordem da derivada a ser utilizada, bem como dos parâmetros do modelo só são possíveis a partir da comparação (calibragem) a partir de dados reais, dados estes que os autores não conhecem no momento.

\section{CONCLUSÕES}

Neste trabalho apresentamos um modelo bastante simples para o compreendimento da dinâmica do ritmo circadiano, para o qual introduzimos as derivadas de ordem fracionária como uma alternativa para descrever efeitos de memória no sistema. A este modelo denominamos de Modelo de Strogatz Generalizado. Embora tenhamos conseguido garantir resutados de boa colocação para o modelo de Strogatz generalizado, não foi possível, neste trabalho, obter de forma explícita a solução do sistema, nem mesmo os pontos de sincronização do modelo, em contraste ao modelo de Strogatz (1)-(2). Tal fato deve-se em partes as complicações impostas pela definição das derivadas de ordem fracionária. Por outro lado, apresentamos resultados numéricos que descrevem de maneira bastante precisa a solução do problema (garantido pelos resultados de precisão do método numérico utilizado). Também percebemos que os resultados numéricos reproduzem os efeitos de memória esperados, fazendo com que os pontos de sincronização dos modelos sejam sentidos de maneia adiantada quando comparados com os resultados o modelo de Strogatz. 
Temos alguns objetivos a serem atingidos em trabalhos futuros. Dentre estes esta a obtenção de dados reais para a calibragem dos parâmetros no modelo e a ordem da derivada, além de obter resultados analíticos para os pontos de sincronização no modelo de Strogatz generalizado.

\section{AGRADECIMENTOS}

N. M. Contessa agradece a CAPES e ao PPGMC pelo suporte parcial. A De Cezaro agradece a FAPERGS via ARD/PPP/FAPERGS/CNPq pelo apoio parcial.

\section{REFERÊNCIAS BIBLIOGRÁFICAS}

1. Strogatz HS. Human sleep and circadian rhythms: a simple model based on two coupled oscillators. Journal of Mathematical Biology. 1987 Jul;(25):327-347, doi: 10.1007/BF00276440.

2. Takahashi JS, Vitaterna MS, Turek FW. Overview of circadian rhythms. Alcohol Research and Health. 2001; 25(2):85-93, doi: 10.1016/B0-72-160797-7/50008-2.

3. Moore-Ede MC, Richardson GS. Medical implications of shift-work. Annual review of Medicine. 1985; 36:607-617, doi: 10.1146/annurev.me.36.020185.0031354.

4. Glaeser SS. Modelagem de sistemas biológicos: Ritmo circadiano [dissertação]. Universidade Federal do Rio Grande, Rio Grande; 2016. 125 p.

5. Pimenv TC, Kelly A, Korobeinikov A. Memory effects in population dynamics: Spread of infectious isease as a case study. Mathematical Modelling of Natural Phenomena. 2012 Jun;(7):206-226, doi: $10.1051 / \mathrm{mmnp} / 20127313$.

6. Oldham KB, Spanier J. The Fractional Calculus: Theory and Applications of Differentiation and Integration to Arbitrary Order. Academic Press. Copyright; 2005. 194 p.

7. Diethelm K. The Analysis of Fractional Differential Equations. Braunschweig. Springer; 2010, Vol. 2004, doi: 10.1007/978-3-642-14574-2.

8. Garrappa R. Short tutorial: Solving fractional differential equations by Matlab codes. Department of Mathematics University of Bari, Italy. 2014 Jun. 